\title{
Excipient Selection for Thermally Stable Enveloped and Non-Enveloped Viral Vaccine Platforms in Dry Powders
}

\author{
Steven P. Toniolo ${ }^{\mathrm{a}}$, Sam Afkhami ${ }^{\mathrm{b}}$, Ahmad Mahmood ${ }^{\mathrm{c}}$, Cécile Fradin ${ }^{\mathrm{c}}$, Brian D. Lichty ${ }^{\mathrm{b}}$, \\ Matthew S. Miller ${ }^{d}$, Zhou Xing ${ }^{\mathrm{b}}$, Emily D. Cranston ${ }^{\mathrm{a}, \mathrm{e}, \mathrm{f}}$, Michael R. Thompson ${ }^{\mathrm{a}^{*}}$ \\ ${ }^{a}$ Department of Chemical Engineering, McMaster University, Hamilton, Ontario, Canada \\ ${ }^{\mathrm{b}}$ McMaster Immunology Research Centre and Department of Pathology \& Molecular Medicine, McMaster University, Hamilton, \\ Ontario, Canada \\ ${ }^{c}$ Department of Physics and Astronomy, McMaster University, Hamilton, Ontario, Canada \\ d Michael G. DeGroote Institute for Infectious Diseases Research, McMaster Immunology Research Centre, and Department of \\ Biochemistry \& Biomedical Sciences, McMaster University, Hamilton, Ontario, Canada \\ e Department of Wood Science, University of British Columbia, 2424 Main Mall, Vancouver, BC, V6T 1Z4, Canada \\ ${ }^{\mathrm{f}}$ Department of Chemical and Biological Engineering, University of British Columbia, 2360 East Mall, Vancouver, BC V6T \\ 1Z3, Canada \\ *Corresponding Author: E-mail address: mthomps@mcmaster.ca (M.R. Thompson)
}

\section{Nomenclature}

$\begin{array}{ll}\text { AdHu5 } & \text { human type } 5 \text { adenovirus } \\ \text { CTC } & \text { controlled temperature chain } \\ \text { DSC } & \text { differential scanning calorimetry } \\ \text { GFP } & \text { green fluorescent protein } \\ \text { MEM } & \text { minimum essential medium } \\ \text { PBS } & \text { phosphate buffered saline } \\ \text { RNA } & \text { ribonucleic acid } \\ \text { SEM } & \text { standard error of the mean } \\ \mathrm{T}_{\mathrm{g}} & \text { glass transition temperature } \\ \mathrm{T}_{\mathrm{m}} & \text { phase transition temperature } \\ \text { VSV } & \text { vesicular stomatitis virus } \\ \text { WHO } & \text { World Health Organization }\end{array}$

1 Abstract

2 Two enveloped viral vectors, vesicular stomatitis virus and influenza virus, and a non-enveloped 3 viral vector, human adenovirus type 5, were encapsulated by spray drying to enhance thermal 4 stability. Results with these candidates led to the hypothesis that stability performance of chosen 
excipients may be less virus-specific, as previously postulated in the literature, and more differentiated based on whether the virus has a lipid envelope. Spray dried samples were characterized for their thermal properties, RNA viability and in vitro viral activity after storage at $37^{\circ} \mathrm{C}$ for up to 30 days or at $45^{\circ} \mathrm{C}$ for up to 3 days. The enveloped viral vectors, as a group, were more thermally stable in trehalose while the non-enveloped viral vector showed higher activity with mannitol as the primary excipient in blends. Trehalose shows strong hydrogen bonds with the envelope's lipid membrane than the other carbohydrates, more effectively replacing water molecules while maintaining the fluidity of the membrane. Conversely, the small size of mannitol molecules was attributed to the more effective hydrogen bonding between water and the protein capsid of non-enveloped viral vectors. In all cases, a matrix with high glass transition temperature contributed to thermal stabilization through vitrification. This work suggests that carbohydrate stabilizer selection may be more dependent on the envelope rather than the specific viral vector, which, if universally true, will provide a guideline for future formulation development.

KEYWORDS: spray drying, enveloped, vitrification, viral, vaccine

\section{Introduction}

The stabilization of vaccines, including viral-based vaccines, through cryopreservation is the current best practice to ensure that stored vaccines maintain their efficacy when administered to humans [1]. This requires vaccines in aqueous media to be kept below $2-8{ }^{\circ} \mathrm{C}$ [1], and often well below this range, resulting in the necessity of a cold chain management system for the production, transportation, and storage of vaccines. The implementation of a cold chain system is costly to suppliers and governments, whereas failure to adhere to management protocols can have serious consequences to public health. As such, the World Health Organization (WHO) has advocated for alternative storage methods to alleviate the dependency on cold chain systems [2]. This has led to the development of thermally stable vaccines to preserve the virus in a dry state based on vitrification $[3,4]$.

Broadly, viruses can be classified into two structural groups, enveloped and nonenveloped. Enveloped viruses, such as vesicular stomatitis virus (VSV) and influenza virus, have their genetic material confined within a protein capsid that is surrounded by a lipid membrane [5]. The genetic material of non-enveloped viruses, like adenovirus, is enclosed within a rigid protein capsid and lacks a lipid membrane [6]. The lipid membrane and protein capsid participate differently in the two relevant mechanisms involved in the preservation of biologics in the dry state (intended to eliminate the dependency for cold chain storage); the two mechanisms are water replacement hypothesis and vitrification theory [7]. The two mechanisms are not mutually exclusive and both are often required to preserve the biologic in the dry state[8]. Damaging these layers in the preparation of dry powder vaccines is generally felt to be a major cause for reduced viral infectivity and vaccine efficacy. 
The water replacement hypothesis considers stabilization of the virus with carbohydrates based on chemical interactions. The modelled mechanism states that upon dehydration, the hydroxyl groups of the carbohydrate, form hydrogen bonds with the protein capsid to maintain the native protein structure and prevent denaturation [9]. Similarly, with lipid membranes, upon dehydration, the hydroxyl groups of the carbohydrate will stabilize phosphate heads of the lipids through the formation of hydrogen bonds [10]. Without the presence of these stabilizing hydrogen bonds, the lipid membrane will experience an increase in van der Waals attraction between the hydrocarbon tails of the lipid bilayer [10], resulting in fusion and increased rigidity of the bilayer, as the membrane transitions into a gel (solid) state [7,11]. The temperature at which this transition occurs is known as the phase transition temperature $\left(T_{m}\right)$, below which a membrane is in its gel state. The $\left(\mathrm{T}_{\mathrm{m}}\right)$ increases when the hydrogen bonds between water and the phosphate heads are not replaced [12]. When the gel state lipid membrane is rehydrated, it locally transitions back to the disordered fluid-like liquid crystalline phase, resulting in nonuniform fluidity and leakage of internal components. The hydroxyl groups of some known sugars, such as sucrose and trehalose, have been shown to be more effectively able to replace the hydrogen bonds formed between water and the phosphate heads upon dehydration of the lipid membrane, stabilizing the phase transition temperature and preserving membrane fluidity and viral function [13].

Vitrification theory is centered around the concept that biologics can remain active through encapsulation by a rigid, amorphous glassy phase of a carbohydrate matrix [14]. An encapsulated biologic is less susceptible to protein denaturation that would otherwise occur during aggregation of the biologics or by thermal stresses. A glassy matrix is ideal for vitrification as its disordered state allows for improved encapsulation of the biologics compared to a crystalline material [15]. Molecular mobility in the glassy amorphous phase governs how well a biologic is encapsulated over time and can be characterized by difference between the storage temperature of a material and its glass transition temperature $\left(\mathrm{T}_{\mathrm{g}}\right)$ [16]. As the storage temperature approaches the material $\mathrm{T}_{\mathrm{g}}$, the molecular mobility of the material increases significantly, which will lead to reduced confinement and ultimately yield protein denaturation [16]. When the storage temperature of a material is $\sim 50{ }^{\circ} \mathrm{C}$ below the $\mathrm{T}_{\mathrm{g}}$, known as the Kauzmann temperature, molecular mobility in the amorphous phase is deemed to be insignificant and is ideal for long-term storage of biologics [17]. The presence of water due to high humidity can depress the $T_{g}$ of many matrix materials, resulting in rapid activity loss $[18,19,20]$, as well as degradation of encapsulated biologics over time [16]. Additionally, low $\mathrm{T}_{\mathrm{g}}$ materials have a greater tendency to recrystallize at ambient temperatures due to higher molecular mobility of the material itself, which is undesirable for vitrification [16].

Lyophilisation is the preferred method for vitrification of biologics in industry, but the process is time consuming, limited by its batch throughput [21] and biologics can be disturbed by stresses endured during the freezing and drying process [22]. An alternative to lyophilisation that has garnered attention in academic and industrial research recently, is the use of spray drying to produce dry powders, with applications as vaccines, for example [1]. The process uses pressurized gas to aerosolize a liquid feed containing the vaccine and excipient formulation, into 85 a heated chamber, where it is then rapidly dried into particles which can be collected in a cyclone 
chamber [23]. Vitrification by spray drying involves undesirable transport phenomena related to droplet formation and drying but offers many demonstrated benefits as well to encapsulate and protect vaccines, including tunability, scalability and relatively short batch time [1].

Recent efforts to improve thermal stability of viral vectors through spray drying, have yielded encouraging results [14]; however, there is a fundamental lack of knowledge on how different viral vectors behave when spray dried with various excipients. The literature in this field has almost exclusively focused on the thermal stability of one biologic at a time and repeatedly concluded that the choice of stabilizing carbohydrate requires broad and substantial screening to yield ideal storage results (normally at $25^{\circ} \mathrm{C}$ for several months or under accelerated evaluation at temperatures closer to $40^{\circ} \mathrm{C}$ for many days). This study examines differences in the thermal stabilization of spray dried viruses that are classified by whether they are enveloped (influenza and VSV) or non-enveloped (human adenovirus type 5; AdHu5). The chosen stabilizing sugar matrices for discussion in this paper were found through broader screening studies to show superior stabilization of the biologics, demonstrated by using identical (nonoptimized) spray drying conditions. The intent of the work is to explain the observed preference of sugar species in stabilizing enveloped versus non-enveloped viral vectors to ultimately provide guidance in excipient selection for this new class of dry powder vaccine products.

\section{Materials and Methods}

\subsection{Chemicals and Viral Vectors}

D-(+)-trehalose dihydrate, D-mannitol, dextran $\left(\mathrm{M}_{\mathrm{r}} 40,000 \mathrm{kDa}\right)$, anhydrous lactose were purchased from Sigma-Aldrich (Ontario, Canada). Culture media was prepared from Alpha Minimum Essential Medium Eagle ( $\alpha$-MEM) (in house according to protocol by the supplier, Life Technologies; Ontario, Canada) with $10 \%$ fetal bovine serum and $1 \%$ streptomycin/penicillin (Invitrogen; Ontario, Canada). Phosphate buffered saline (PBS) was prepared in house.

Recombinant replication-deficient human type 5 adenovirus expressing green fluorescent 114 (VSVGFP) were produced in the vector facility of the McMaster Immunology Research Centre 115 (MIRC), as described previously [24]. The A/Puerto Rice/8/1934 H1N1 mNeon-2A-HA virus 116 (Influenza mNeon) was a gift from Dr. Heaton of Duke University and was propagated in 117 embryonated chicken eggs [25]. From this point on, these viruses are termed AdHu5, VSV and 118 influenza for brevity.

\section{$119 \quad 2.2 \quad$ Spray Drying of Viral Vectors}

Spray dried powder viral vectors were produced by spray drying using a Mini Spray 121 Dryer B-290 (Büchi; Switzerland) with $0.7 \mathrm{~mm}$ spray nozzle and high performance cyclone 122 attachment, as previously described [18]. For all spray dried excipients, the spray dryer was 123 operated at the same (non-optimized) conditions, namely a spray gas flow rate of $439 \mathrm{~L} / \mathrm{h}$, feed 
124 solution of $234 \mathrm{~mL} / \mathrm{h}$ and a nozzle inlet temperature of $110{ }^{\circ} \mathrm{C}$. The concentration of all feed 125 solutions was $4 \mathrm{wt} \%$ solute.

\section{$126 \quad 2.3 \quad$ Storage of the Powder Viral Vector}

Samples of the dry powder viral vectors were stored individually in closed $2 \mathrm{~mL}$ Nalgene General Long-Term Storage Cryogenic Tubes (Nalgene; Ontario, Canada) sealed with Parafilm Wax (Bemis NA; Wisconsin, US). The tubes were placed in a re-sealable plastic bag and stored within a glass jar filled with gel desiccant and sealed with Parafilm Wax to ensure a low humidity environment $(<10 \% \mathrm{RH})$. The glass jars were sealed within another resealable plastic bag and placed in a water bath for temperature control at $37^{\circ} \mathrm{C}$ for up to 30 days or under "accelerated storage conditions" at $45^{\circ} \mathrm{C}$ for up to 3 days. The liquid control containing only PBS buffer and AdHu5, VSV, or influenza were stored in a plastic resealable bag within a tightly sealed glass jar without gel desiccant.

\subsection{Differential Scanning Calorimetry (DSC)}

Thermograms for the spray dried powders (without viral vector) were measured by differential scanning calorimetry (DSC). Powder samples were weighed out between 4-8 mg and hermetically sealed in Tzero $^{\mathrm{TM}}$ pans. The samples were analyzed with a Q200 Differential Scanning Calorimeter (TA Instruments; New Castle, DE). Samples were tested using a modulated DSC protocol from $20^{\circ} \mathrm{C}$ to $200{ }^{\circ} \mathrm{C}$ at a ramp rate of $1{ }^{\circ} \mathrm{C} / \mathrm{min}$, an amplitude of $\pm 1^{\circ} \mathrm{C}$ and an oscillation period of $30 \mathrm{~s}$, under a nitrogen purge gas flow rate of $50 \mathrm{~mL} / \mathrm{min}$. The glass transition temperature $\left(\mathrm{T}_{\mathrm{g}}\right)$ of the sample was determined using TA Universal Analysis software

\subsection{RNA Staining}

Spray dried samples of VSV were stored, as described above, under the accelerated storage conditions for 3 days at $45{ }^{\circ} \mathrm{C}$. The samples were reconstituted with nuclease free water. A liquid control of VSV in PBS was dried and stored under the same conditions. The RNA staining was conducted in an opaque flat bottom 96 well plate with the QuantiFluor RNA System (Promega; Madison, WI) in accordance with the Promega QuatiFluor RNA System Protocol [26]. Fluorescence was measured using a Spectramax i3 (Molecular Devices; San Diego, CA). To mitigate the effects of the different excipients on the fluorescence readout, standard curves were prepared for each excipient or blend of excipients at the same concentration of the reconstituted spray dried samples.

\subsection{FTIR of Lipid-Sugar Interactions}

\subsubsection{POPC- Sugar Liposome Preparation}

To mimic the lipid-sugar interactions that occur between the lipid envelope of an enveloped virus and the encapsulating sugar, a lipid analogue was selected and assembled into

159 liposomes and mixed with the sugar excipients. The selected lipid was 1-palmitoyl-2-oleoyl-sn160 glycero-3-phosphocholine (POPC) from Avanti Polar Lipids, Inc. (Alabaster, AL) as POPC is 161 found in relatively large percentages in the cellular and viral envelope [5]. POPC was preferred 
over lipid extracts from the viral envelope due to the large quantity of lipid needed to create liposomes with high lipid-to-sugar ratios for each sugar compound, to elucidate their interactions

164 with the lipid.

165 The lipids were first prepared into lipid films by evaporating chloroform dissolved lipids under 166 an argon gas stream and dried further in a vacuum chamber overnight. After drying, the films 167 were rehydrated with MilliQ water $(18.2 \mathrm{M} \Omega \mathrm{cm})$ to a concentration of $10 \mathrm{mg} / \mathrm{mL}$ and mixed 168 with the respective excipient blend $(10 \mathrm{mg} / \mathrm{mL})$ to the desired lipid:sugar ratio by mass. The solution was vortexed and subjected to 10 freeze-thaw cycles using liquid nitrogen and a warm water bath, to give unilamellar liposomes, with sugar present both inside and outside of the liposome. The solution was then extruded 11 times through a $100 \mathrm{~nm}$ diameter pore membrane using the Avanti ${ }^{\circledR}$ Mini-Extruder (Avanti Polar Lipids, Inc. Alabaster, AL), to create a more monodisperse liposome size. Dynamic light scattering (DLS) analysis of the POPC-sugar solution with a Malvern ZetaSizer Nano (Malvern Panalytical; Montreal, QC) yielded an average particle size of approximately $140 \mathrm{~nm}$. The liposome suspension was transferred to a vial, purged with argon gas, wrapped in parafilm, and stored at $4{ }^{\circ} \mathrm{C}$. Suspensions were used within 177 one week [27].

178

\subsubsection{POPC-Sugar Preparation for FTIR Sampling}

Spectroscopy-grade $\mathrm{KBr}$ powder (Sigma-Aldrich; Mississauga, $\mathrm{ON}$ ) was added to the POPC-sugar liposomes at a ratio of $25 \mu \mathrm{L}$ of liposome $/ 100 \mathrm{mg} \mathrm{KBr}$ to allow for the sample to be handled in the FTIR while ensuring a strong signal. The KBr-liposome mixture was dried under vacuum in a chamber containing desiccant for 4 hours to remove the bulk water. Residual water was removed by drying the sample in an oven at $110^{\circ} \mathrm{C}$ overnight.

\subsubsection{Fourier Transform Infrared Spectroscopy (FTIR)}

Infrared absorption measurements were carried out using a Nicolet 6700 FT-IR Spectrometer (Thermo Scientific, Waltham, MA) equipped with a Smart iTR Diamond ATR and a DTGS $\mathrm{KBr}$ detector. The acquisition parameters were: $4 \mathrm{~cm}^{-1}$ resolution, 128 co-added interferograms covering a $4000-525 \mathrm{~cm}^{-1}$ wavenumber range at room temperature and the ATR correction feature of the software was applied. Spectral analysis was carried out with the OMNIC Spectra software. The spectral region between 3600 and $3000 \mathrm{~cm}^{-1}$ was selected to measure the $-\mathrm{OH}$ stretching band $(\mathrm{vOH})$ and the band position was calculated as the average of spectral positions of $80 \%$ of the maximum peak height, similar to Wolkers et al. [28].

\subsection{In Vitro Testing of Viral Vectors}

\subsubsection{Cell Culturing}

Vero cells isolated from kidney epithelial cells and A549 lung epithelial cells were thawed from liquid nitrogen and cultured with Alpha Minimum Essential Medium Eagle $(\alpha-$ MEM) in T150 culture flasks. Cell culturing was completed in a humidified Forma Series II Water Jacketed $\mathrm{CO}_{2}$ Incubator (Thermo Scientific Corporation; Waltham, MA) at $37^{\circ} \mathrm{C}$ and 
$5.0 \% \mathrm{CO}_{2}$. When cells appeared to be $80-90 \%$ confluent, they were split into a new T150 culture flask and/or plated in a 96-well plate for in vitro testing.

\subsubsection{GFP Detection and Endpoint TCID Calculation}

The viral activity after the spray drying process and subsequent storage was determined by an endpoint dilution in a 96 flat bottom well plate. For the VSV vector, plated Vero cells were infected with $4 \mathrm{mg}$ of powder (initial concentration of $1.30 \times 10^{9} \mathrm{pfu} / \mathrm{g}$ ) reconstituted in culture media. For influenza, plated Vero cells were infected with $4 \mathrm{mg}$ of powder (initial concentration of $2.51 \times 10^{5} \mathrm{pfu} / \mathrm{g}$ ) reconstituted in culture media. For AdHu5, plated A549 cells were infected with $4 \mathrm{mg}$ of powder (initial concentration of $2.71 \times 10^{8} \mathrm{pfu} / \mathrm{g}$ ). After the overnight incubation of plated the respective cells, the cell media was removed and replaced with an eight-fold serial dilution from each reconstituted sample at a volume of $100 \mu \mathrm{L}$ per well and a total of 4 wells per sample. After overnight incubation, viral infectivity was detected by the presence of a GFP expression indicated by a bright green hue to cells, observed under an EVOS FL Cell Imaging System with a GFP filter (Thermo Scientific Corporation; Waltham, MA). A positive GFP response of a single cell within a well constituted a positive infection response with respect to the endpoint dilution. If greater than $50 \%$ of the wells in the row were non-expressive, the dilution was determined to have reached its endpoint and the median tissue culture infections dose $\left(\mathrm{TCID}_{50}\right)$ was calculated using the Reed-Muench method [29]. Using the Poisson distribution, the results were multiplied by a factor of 0.69 to convert to an approximated pfu value and reported as a viral activity loss of pfu/g of material with error bars calculated as the standard error $(n=3)$. Due to the different starting titres of the various types of viral vectors, the data was plotted as Relative Activity to normalize the activity data between the different viral vectors. Relative Activity was calculated according to Equation 1:

\section{Results}

\subsection{Thermal Storage Properties of Spray Dried Powders}

Spray dried excipients (with no virus included) were stored in the same low relative humidity environment at $37^{\circ} \mathrm{C}$ as the spray dried viral vectors, to investigate changes in glass transition temperature over time; the chosen excipients were selected for an inhalable formulation, and tested individually or as binary blends. The glass transition temperatures of the samples were measured using DSC (Table 1) with an estimated uncertainty in the measurement of $\pm 0.1{ }^{\circ} \mathrm{C}$. Ideal formulations should exhibit an amorphous phase with a glass transition above $100{ }^{\circ} \mathrm{C}$ to ensure normal storage conditions are far below the Kauzmann temperature.

\section{Table 1: Glass transition temperatures $\left(T_{g}\right)$ of excipients after spray drying (Day 0), and after} storage for 7,10 and 15 days at $37^{\circ} \mathrm{C}$ at low relative humidity.

\begin{tabular}{|c|c|c|c|c|}
\hline Sample & $\begin{array}{c}\mathbf{T}_{g}(\text { Day 0, 37 } \\
\left.{ }^{\circ} \mathbf{C}\right)\end{array}$ & $\begin{array}{c}\mathbf{T}_{g}(\text { Day 7, 37 } \\
\left.{ }^{\circ} \mathbf{C}\right)\end{array}$ & $\begin{array}{c}\mathbf{T}_{g}(\text { Day 10, 37 } \\
\left.{ }^{\circ} \mathbf{C}\right)\end{array}$ & $\begin{array}{c}\mathbf{T}_{g}(\text { Day 15, } \\
\left.\mathbf{3 7}^{\circ} \mathbf{C}\right)\end{array}$ \\
\hline
\end{tabular}




\begin{tabular}{|c|c|c|c|c|}
\hline Trehalose & 110 & 98 & 72 & 58 \\
\hline Trehalose/Dextran (1:3) & 88 & 81 & 86 & 88 \\
\hline Trehalose/Dextran (3:1) & 88 & 87 & 67 & 61 \\
\hline Mannitol/Dextran (2:1) & 113 & 109 & 101 & 99 \\
\hline Dextran & 125 & 123 & 113 & 107 \\
\hline
\end{tabular}

Both the dextran and mannitol/dextran particles exhibited the highest $\mathrm{T}_{\mathrm{g}}$ values after

\subsection{Activity of Spray Dried Viral Vectors after 30 Days Storage}

Using the same excipients reported in Table 1, spray dried powders containing AdHu5, VSV and influenza were subjected to storage at $37{ }^{\circ} \mathrm{C}$ in low relative humidity for up to 30 days to evaluate thermal stability. The measured activity losses (reported as relative activity, Eqn. 1) were high due to the non-optimized (yet consistent) spray drying conditions used, but it was decided the study would better reveal excipient effects on the viral vector activity if the process environment was not varied while the sugar stabilizers were changed. spray drying, measured at $125^{\circ} \mathrm{C}$ and $113^{\circ} \mathrm{C}$, respectively. Trehalose spray dried samples had a similarly high, $\mathrm{T}_{\mathrm{g}}$ of $110^{\circ} \mathrm{C}$. The trehalose/dextran ratios of $1: 3$ and $3: 1$ had identical $\mathrm{T}_{\mathrm{g}}$ values after spray drying $\left(88^{\circ} \mathrm{C}\right)$ and were the lowest of the excipients tested. After 10 days of storage at $37{ }^{\circ} \mathrm{C}$, trehalose and $3: 1$ trehalose/dextran exhibited a significant drop in $\mathrm{T}_{\mathrm{g}}$, and became matrices with the lowest $\mathrm{T}_{\mathrm{g}}$ in the study at $72{ }^{\circ} \mathrm{C}$ and $67{ }^{\circ} \mathrm{C}$, respectively. The $\mathrm{T}_{\mathrm{g}}$ is known to decrease because of the gradual plasticization effect of water on carbohydrates which leads to increased molecular mobility of the amorphous phase; however, suitable excipients will experience a smaller change in $\mathrm{T}_{\mathrm{g}}$ over time than poorer candidates. While low, these stored $\mathrm{T}_{\mathrm{g}}$ values still reasonably met the Kauzmann temperature for targeted room temperature storage (but not at $37^{\circ} \mathrm{C}$ ). The $\mathrm{T}_{\mathrm{g}}$ of both trehalose and 3:1 trehalose/dextran continued to decrease slightly to $58{ }^{\circ} \mathrm{C}$ and $61{ }^{\circ} \mathrm{C}$ after 15 days of storage, respectively. The $\mathrm{T}_{\mathrm{g}}$ of mannitol/dextran and dextran samples both dropped by $12{ }^{\circ} \mathrm{C}$ after 10 days of storage but remained above the desired threshold of $100{ }^{\circ} \mathrm{C}$. The $1: 3$ trehalose/dextran blend yielded the smallest change in $\mathrm{T}_{\mathrm{g}}$ after 10 days of storage, barely decreasing from $88^{\circ} \mathrm{C}$ to $86^{\circ} \mathrm{C}$.

(2)

Figure 1 shows the liquid control samples reached a state with no detectable viral activity over the course of the storage study. The decline in activity was greatest for influenza, followed by VSV and lastly, AdHu5. Conversely, the spray dried powders showed superior thermal stability, with some samples retaining up to $50 \%$ of their original titre by the end of the storage period. Titre losses were notably different for the three vectors with the different excipients; however, VSV and influenza showed similar trends across the group of excipients. Activity after storage for 30 days at $37{ }^{\circ} \mathrm{C}$ resembled the activity from an accelerated storage study at $45^{\circ} \mathrm{C}$ after 3 days and accelerated studies also indicated that excipient blends all outperformed individual excipients, except for trehalose (data not shown). 
More specifically, for the spray dried powders with AdHu5, mannitol/dextran exhibited the lowest activity losses and was the only formulation for this viral vector to retain activity after 30 days of storage at $37{ }^{\circ} \mathrm{C}$, with one sample in the set of triplicates yielding no detectable activity; we have previously reported this formulation as ideal for both AdHu5 and recombinant replication-defective chimpanzee type 68 adenovirus [30], which is mentioned to highlight the formulation is not just uniquely suited to one adenovirus. Dextran was considered the least effective excipient at stabilizing AdHu5 since spray dried samples lost most of their activity after only one day of storage whereas the control was completely inactive after 15 days of storage. By Day 30, all samples except for mannitol/dextran were determined to have lost all detectable activity.

Better performing formulations with VSV were trehalose or trehalose/dextran (3:1), both were able to maintain some activity even after 30 days of storage contrary to the other spray Negligible activity was seen for the liquid control after 15 days of storage. Activity losses for samples of trehalose and trehalose/dextran (3:1) appeared to reach a plateau after 10 days. The titre loss of the liquid control was minor initially but exceeded the trehalose and trehalose/dextran samples after 7 days of storage at $37{ }^{\circ} \mathrm{C}$. Dextran was the least effective stabilizer with VSV as well, when considering both spray drying (process loss at Day 0) and storage results together, while the mannitol/dextran blend experienced the greatest loss in activity immediately after the spray drying process.

The best formulation for stabilizing influenza was trehalose, followed by trehalose/dextran (3:1) - similar to the results with VSV. By 15 days of storage, the trehalose formulation was the only influenza sample that retained some viral activity, as all other formulations were below the limits for activity detection. After 30 days, only one out of three samples of the trehalose formulation was able to elicit a positive infection response. In comparison, the liquid control for influenza lost all activity after 10 days of storage and the mannitol/dextran blend yielded the highest initial activity loss (i.e. process loss). The initial starting titre of the influenza vector was approximately 3.0 log lower than either AdHu5 or VSV, and as such activity losses appeared to be relatively greater.

\subsection{Quantification of Intact RNA after Storage of VSV-containing Powders}

To gain a detailed understanding of the damage occurring to the lipid membrane of enveloped viruses during the preparation and storage of dry powder vaccines, the amount of intact RNA after storage was measured through a staining method, using the enveloped VSV as the model system in this case. Samples were tested immediately after spray drying and after three days of accelerated storage at $45{ }^{\circ} \mathrm{C}$ under low relative humidity, and were compared to VSV dried directly from the liquid control (i.e., buffer solution) then aged following the same protocol. The amount of intact RNA after spray drying and storage is shown in Figure 2, exhibiting an inverse correlation (in general) with the activity values shown in Figure 1. With low RNA values, the RNA of the viral vector is exposed and unprotected by the excipient resulting in a lower activity. 
[Figure 2]

The larger intact RNA values in Figure 2 of samples exposed to the spray drying/reconstitution/ageing steps indicates that the encapsulating sugars were able to protect the viral genetic material and prevent damage to the lipid membrane. Exposed RNA denatures quickly and becomes undetectable by the fluorescent dye used in this assay. (Evidence that exposed RNA denatures under these storage conditions and is not detected by the current dye was supported by an additional control experiment conducted whereby stock RNA at $50 \mathrm{ng}$ of $\mathrm{RNA} / \mu \mathrm{L}$ was dried and tested; after 3 days at $45^{\circ} \mathrm{C}$ the detectable RNA decreased $65 \%$ to $17 \pm 2$ ng of RNA/ $\mu \mathrm{L}$.)

The VSV-mannitol/dextran powder had the lowest concentration of detectable RNA at $2.0 \pm 0.4 \times 10^{-6} \mathrm{ng} / \mathrm{pfu}$ after spray drying and also had the lowest detectable RNA after three days of storage $\left(0.6 \pm 0.2 \times 10^{-6} \mathrm{ng} / \mathrm{pfu}\right)$ indicating poor stabilizing performance with VSV. The VSV-trehalose sample had a high value initially and experienced the smallest decrease in detectable RNA over the three days of storage, showing that most RNA was protected. The VSV-dextran powder also exhibited a high RNA value initially but then had the most significant rate of decline (from $6.1 \pm 0.3 \times 10^{-6} \mathrm{ng} / \mathrm{pfu}$ to $1.04 \pm 0.08 \times 10^{-6} \mathrm{ng} / \mathrm{pfu}$ after three days) which suggests that the matrix could not prevent RNA leakage and denaturation during storage. A dried VSV-only control (no excipient) yielded the lowest detectable RNA concentration of all samples after 3 days of storage with only $0.37 \pm 0.01 \times 10^{-6} \mathrm{ng} / \mathrm{pfu}$ measured.

\subsection{Strength of Lipid-Sugar Interactions}

To understand the strength of hydrogen bonding between the lipid membrane analogue (POPC liposomes) and sugar excipients, the average position of the hydroxyl vibrational stretching band $(\mathrm{vOH})$ of the sugar was measured by FTIR. The $v \mathrm{OH}$ position of the different liposome-sugar mixtures as a function of changing the POPC to sugar mass ratio is displayed in Figure 3.

[Figure 3]

Hydrogen bonding interactions between the lipids and sugars became more apparent in this test as the POPC:sugar ratio increased, i.e., at increasing ratios, the lipid-sugar hydrogen bonding dominated over the sugar-sugar hydrogen bonding. A decrease in the $\mathrm{vOH}$ peak position indicates a shorter hydrogen bond length, which means a stronger bond. We believe that at higher POPC:sugar ratios, the test was better at approximating what happens at the excipient-viral vector envelope interface and focus the discussion primarily on the 3:1 POPC:sugar data points. The excipients that form the strongest hydrogen bonds with lipids thus follow the order trehalose>trehalose/dextran 3:1>mannitol:dextran 3:1>trehalose/dextran 1:3, according to Figure 3. This trend generally matches the activity (particularly at day 5 in Figure 1) and RNA viability, implying that hydrogen bond strength is linked to the ability of certain sugars to thermally stabilize viral vectors with lipid membrane envelopes. 
More specifically for trehalose and trehalose/dextran 3:1 formulations, the $\mathrm{vOH}$ band position decreased with increasing POPC:sugar ratio, indicating increasing hydrogen bond strength and strong lipid-sugar interactions. Conversely, the $\mathrm{vOH}$ band position for mannitol/dextran and trehalose/dextran 1:3 increased with increasing POPC:sugar ratio, indicating that these sugar blends do not contribute significantly to hydrogen bonding with the lipids meaning that they do not be expected to protect the envelope from leaking upon rehydration. Similarly, Wolkers et al. demonstrated that mixing trehalose, glucose and sucrose with POPC vesicles led to a decrease in band position and stronger hydrogen bonding whereas dextran did not [28]. The inability for dextran to hydrogen bond significantly with POPC was attributed to its larger, branched molecular structure [28,31] and explains why the excipient blend with the higher concentration of dextran (trehalose/dextran 1:3) showed weaker hydrogen bonding abilities in the test. Like dextran, other polysaccharide glucans have also been shown to

\section{Discussion}

Spray drying has emerged to be a new technology for vaccine formulation development. It is growing apparent that single excipient formulations lack sufficient performance in storage testing to be considered ideal matrices. It is therefore contingent upon the community to develop guidance in formulating appropriate mixtures that reduce the development window for dry powder vaccine manufacture. In the present study, it was evident through the activity storage data that the same excipient formulations did not thermally stabilize all viral vectors equivalently when spray dried. The scope of this first study on the theory is obviously too small in the number of viral vectors examined to make definitive assertions but we have seen similar preferential stabilization by the same sugars with other viral vectors during preliminary testing in our lab to conclude that the trends merit serious consideration. The best performing excipients to be used in the spray drying of VSV and influenza viral vectors were trehalose and trehalose/dextran (3:1), based on storage data, showing similarities between the two enveloped viral vectors. The best performing spray dried formulation for AdHu5, the non-enveloped viral vector, in terms of storage data was mannitol/dextran, which conversely experienced the greatest activity loss when spray dried with VSV and influenza. The same mannitol/dextran blend demonstrated excellent stabilization of a chimpanzee type 68 adenovirus in previously reported results by the authors as well [30]. All of these viral vectors are among the important candidate vaccine platforms against infectious diseases and cancer.

Differences in thermal stabilization appear attributable to the vitrifying properties of the sugar excipients and the chemical interactions (or lack thereof) between the sugars and the outer surfaces of the viral vectors. The balance of considerations mentioned below for dextran make apparent that excipient selection is quite complicated but not without understandable rules. The use of dextran in the studied blends was universally favored by both enveloped and nonenveloped viral vectors due to its high $\mathrm{T}_{\mathrm{g}}$ and its ability to minimize $\mathrm{T}_{\mathrm{g}}$ depression over time, as indicated in Table 1. Since dextran is a large molecule, it has low molecular mobility and as such, movement of the virus within the matrix should be limited. This limitation in movement 
should ensure that the virus remains encapsulated and does not aggregate, which would otherwise result in a loss of activity [16]. However, alone, dextran in spray dried samples demonstrated poor activity retention for both enveloped and non-enveloped viruses (Figure 1f). The use of dextran as the only excipient was considered to be non-ideal, as its large size and branching makes it unable to closely encapsulate the vaccine or prevent aggregation, and unable to effectively replace water hydrogen bonds [28,31] to minimize membrane leakage (Figure 2) resulting in reduced activity as shown previously for encapsulated protein systems [33]. Furthermore, dextran in moderate to high concentrations within a blend yielded high activity losses for enveloped viruses as observed with the trehalose/dextran (1:3) blend for VSV and influenza (Figure 1d). This was further exemplified by the overall increase of the $v \mathrm{OH}$ position of trehalose/dextran (1:3) with increasing lipid:sugar ratios, indicating weaker hydrogen bonding with the lipid membrane [28,32], as shown in Figure 3.

When dextran was used at lower concentrations in conjunction with smaller carbohydrates, such as mannitol and trehalose, its dominant function was to limit molecular mobility within the matrix (Table 1). The smaller sugars then had the potential to directly interact with the viral vectors, bridging the virus with the stabilizing matrix and minimizing activity loss [33]. Again, the increase in $\mathrm{vOH}$ peak position at higher lipid:sugar ratios (Figure 3), indicates that the trehalose/dextran (1:3) blend was not able to interact with the envelope analogue via hydrogen bonding as strongly as the trehalose/dextran (3:1) blend and trehalose-only formulations. Thus, while dextran is an attractive excipient for thermal stabilization of vaccines due to its high $\mathrm{T}_{\mathrm{g}}$, its inability to stabilize lipid membranes through physical/chemical bonding makes it a poor primary material for stabilizing enveloped viral vectors and as such, should be used as a secondary excipient in blends at low relative concentrations.

Trehalose and its blends were generally found to be good thermal stabilizers for all spray dried viral vectors tested here, but more specifically preferred for the enveloped vectors. This agrees with other studies reported in the literature showing trehalose to be a good stabilization agent, known to effectively replace water molecules surrounding phosphate heads in lipid membranes upon dehydration, satisfying the water replacement hypothesis $[7,34,35]$. Trehalose is able to maintain the $T_{m}$ of a lipid membrane upon dehydration, which is necessary to stabilize enveloped viral vectors [36] and prevent leakage of viral genetic material through a damaged lipid membrane. This was supported here by the larger amounts of intact RNA and strong hydrogen bonding measured for trehalose-rich systems (Figure 2 and 3, respectively). For a disaccharide, trehalose possesses a relatively high $\mathrm{T}_{\mathrm{g}}$ and forms a primarily amorphous glassy phase when spray dried [17], vitrifying the viral vector. However, the depression of the $T_{g}$ of trehalose during storage at elevated temperatures seen in Table 1 suggests that the sugar is not without negative performance factors. The formed matrix experiences significant plasticization over time [16] such that the storage temperature was below the Kauzmann temperature (by the $10^{\text {th }}$ day) whereby the matrix experiences significant molecular mobility. This depression could be due to residual moisture after spray drying as trehalose is known to be a hygroscopic material [37], though residual moisture testing was outside of the scope of this project. Overall, the decrease in $\mathrm{T}_{\mathrm{g}}$ for trehalose-containing formulations correlated with a decrease in viral activity (Figure 1b-d), particularly over the first 10 days after which a plateau was reached suggesting 
that the molecular mobility of the matrix stopped, changing as the moisture content (and $\mathrm{T}_{\mathrm{g}}$ ) reached equilibrium [16]. These results imply that the water replacement mechanism dominates over the vitrification mechanism for enveloped viral vector stabilization.

While trehalose was able to retain activity of spray dried AdHu5 and outperformed the liquid control, the mannitol/dextran blend was significantly better even after 30 days of storage at $37^{\circ} \mathrm{C}$ (Figure 1e) with this non-enveloped vector. The mannitol/dextran blend was best at minimizing activity loss of AdHu5 during storage due to the high $\mathrm{T}_{\mathrm{g}}$ of the blend and the ability of mannitol to replace hydrogen bonds with the protein capsid of the non-enveloped viral vector [38]. On the other hand, mannitol is not an ideal excipient, especially for enveloped viral vectors, because it readily crystallizes [38] which could lead to crystal nucleation in the dry state and pierce the lipid membrane of enveloped viruses [39]. Polyols like mannitol, can also alter the phase of a lipid membrane from a bilayer to a hexagonal II phase, resulting in leakage [40]. Once the lipid membrane is perturbed, the genetic material of the virus will leak out and lower the infectivity of the viral vector. The decrease in detectable RNA between 0 and 3 days of storage for all excipients with VSV (Figure 2) is an indicator that RNA has likely leaked through the membrane and is unprotected on the outside of the virus. The VSV-mannitol/dextran particles had the lowest concentrations of intact RNA (both immediately after spray drying and after 3 days of storage), corresponding to large activity losses in vitro.

After analyzing the trends between the spray dried formulations with AdHu5, VSV, and influenza a set of general criteria can be identified for the production of thermally stabilized enveloped or non-enveloped viral vectors. For dry powder vaccines based on a non-enveloped viral platform, a glassy matrix with high $\mathrm{T}_{\mathrm{g}}$ is preferred that has minimal molecular mobility and encapsulates the virus. However, mobility increases with time due to plasticization and as such, materials that maintain their $\mathrm{T}_{\mathrm{g}}$ over time are ideal. These material characteristics can be achieved through the blending of small monosaccharides (mannitol) as a primary component, with branched polysaccharides (dextran) in lower concentrations. The monosaccharides are not limited by steric effects and will tightly encapsulate the non-enveloped viral vector, while the polysaccharide will behave as a stabilizing matrix to enhance and maintain the $\mathrm{T}_{\mathrm{g}}$ of the blend. Stabilization of enveloped viruses similarly requires a glassy matrix with high $\mathrm{T}_{\mathrm{g}}$, but the formulation must also contain sugars that have demonstrated the ability to form strong hydrogen bonds with the phosphate heads of lipid membranes to maintain fluidity of the lipid bilayer and prevent a phase transition to the gel state. To achieve this desired effect, polysaccharides (dextran) in lower concentrations can be used within the excipient blend to form a glassy matrix with high $\mathrm{T}_{\mathrm{g}}$. However, the steric effects of the polysaccharide impede the stabilization of individual phosphate heads of the viral lipid bilayer, resulting in a loss of fluidity and viral activity when used alone. The addition of small branched disaccharides (trehalose) as the primary component in a blend is necessary to form the hydrogen bonds via its hydroxyl groups, and maintain the $T_{m}$ of the lipid bilayer, protecting the viral envelope. Thus, when selecting excipients for stabilization of enveloped viruses in the dry state, both the $T_{g}$ of the sugar and the 


\section{Conclusion}

This study shows that there are differences in the thermal stabilization of enveloped and non-enveloped viral vectors when spray dried with the same formulation. When selecting excipients it is imperative to consider the viral structure. Trehalose and trehalose/dextran (3:1) were the best excipients at retaining viral activity when spray dried with enveloped viral vectors (VSV and influenza) and mannitol/dextran blends retained the most activity for the nonenveloped viral vector (AdHu5). Conversely, mannitol/dextran was the least successful at stabilizing the VSV and influenza vectors. A set of model experiments with lipid liposomes and sugar mixtures supported the importance of hydrogen bonding as a primary mechanism in stabilizing enveloped viral vectors. Furthermore, RNA staining of spray dried powders implied that excipients that did not hydrogen bond with the viral vector envelope allowed for leakage and exposure of the viral genetic material and only small quantities of intact RNA were detectable after storage. The thermal stabilization mechanism for non-enveloped viral vectors was dominated by matrix morphology although the ability for the matrix to replace water bonds with the protein capsid can also improve the stabilization. This work contributes new design rules for

\section{Acknowledgements}

489 The authors thank Dr. Xueya Feng for viral vector production, Dr. Nicholas Heaton (Duke 490 University) for providing the influenza mNeon viral vector and Daniel Osorio for DLS 491 measurements. The authors also acknowledge the Biointerfaces Institute at McMaster University 492 for use of their facilities. This study is supported by funds from the Quebec Consortium for 493 Drug Discovery (CQDM), Ontario Centres of Excellence (OCE), the Canadian Institutes of 494 Health Research, and Natural Sciences and Engineering Research Council of Canada. Cranston 495 holds the Canada Research Chair in Bio-based Nanomaterials (Tier 2). Miller holds a CIHR New Investigator Award and and Early Researcher Award from the Government of Ontario. 


\section{References}

[1] G. Kanojia, G.-J. Willems, H. W. Frijlink, G. F. A. Kersten, P. C. Soema, and J.-P. Amorij, "A Design of Experiment approach to predict product and process parameters for

[6] M. O. Lasaro and H. C. Ertl, "New Insights on Adenovirus as Vaccine Vectors," Mol. a spray dried influenza vaccine," Int. J. Pharm., vol. 511, no. 2, pp. 1098-1111, 2016.

[2] World Health Organisation, "The controlled temperature chain ( CTC ): frequently asked questions," 2014.

[3] D. T. Brandau, L. S. Jones, C. M. Wiethoff, J. Rexroad, and C. R. Middaugh, "MINIREVIEW Thermal Stability of Vaccines," J. Pharm. Sci., vol. 92, no. 2, pp. 218231, 2003.

[4] J. Huang et al., "A novel dry powder influenza vaccine and intranasal delivery technology: Induction of systemic and mucosal immune responses in rats," Vaccine, vol. 23, no. 6, pp. 794-801, 2004.

[5] P. T. Ivanova, D. S. Myers, S. B. Milne, J. L. Mcclaren, P. G. Thomas, and H. A. Brown, "Lipid Composition of the Viral Envelope of Three Strains of In fl uenza Virus - Not All Viruses Are Created Equal,” 2015. Ther., vol. 17, no. 8, pp. 1333-1339, 2009.

[7] J. H. Crowe, J. F. Carpenter, and L. M. Crowe, "the Role of Vitrification in Anhydrobiosis," Annu. Rev. Physiol., vol. 60, pp. 73-103, 1998.

[8] D. K. Hincha, A. V. Popova, and C. Cacela, "Effects of Sugars on the Stability and Structure of Lipid Membranes During Drying," Adv. Planar Lipid Bilayers Liposomes, vol. 3, no. 5, pp. 189-217, 2006.

[9] M. A. Mensink, H. W. Frijlink, K. van der Voort Maarschalk, and W. L. J. Hinrichs, "How sugars protect proteins in the solid state and during drying (review): Mechanisms of stabilization in relation to stress conditions," Eur. J. Pharm. Biopharm., vol. 114, pp. 288295, 2017.

[10] J. H. Crowe, L. M. . C. Crowe, and D. Chapman, "Preservation of Membranes in Anhydrobiotic Organisms : The Role of Trehalose," Science (80-. )., vol. 223, no. 4637, pp. 701-703, 1984.

[11] E. A. Golovina, A. Golovin, F. A. Hoekstra, and R. Faller, "Water replacement hypothesis in atomic details: Effect of trehalose on the structure of single dehydrated POPC bilayers," Langmuir, vol. 26, no. 13, pp. 11118-11126, 2010.

[12] J. H. Crowe, L. M. Crowe, J. F. Carpenter, and C. Aurell Wistrom, "Stabilization of dry phospholipid bilayers and proteins by sugars.," Biochem. J., vol. 242, no. 1, pp. 1-10, 1987.

[13] J. P. Amorij, A. Huckriede, J. Wilschut, H. W. Frijlink, and W. L. J. Hinrichs, "Development of stable influenza vaccine powder formulations: Challenges and 
possibilities," Pharm. Res., vol. 25, no. 6, pp. 1256-1273, 2008.

[14] L. Chang et al., "Mechanism of protein stabilization by sugars during freeze-drying and storage: Native structure preservation, specific interaction, and/or immobilization in a glassy matrix?," J. Pharm. Sci., vol. 94, no. 7, pp. 1427-1444, 2005.

[15] V. Saluja, J. P. Amorij, J. C. Kapteyn, A. H. de Boer, H. W. Frijlink, and W. L. J. Hinrichs, "A comparison between spray drying and spray freeze drying to produce an influenza subunit vaccine powder for inhalation," J. Control. Release, vol. 144, no. 2, pp. 127-133, 2010.

[16] B. C. Hancock, S. L. Shamblin, and G. Zografi, "Molecular Mobility of Amorphous Pharmaceutical Solids Below Their Glass Transition Temperatures," Pharm. Res. An Off. J. Am. Assoc. Pharm. Sci., vol. 12, no. 6, pp. 799-806, 1995.

[17] S. Hoe et al., "Use of a fundamental approach to spray-drying formulation design to facilitate the development of multi-component dry powder aerosols for respiratory drug delivery.," Pharm. Res., vol. 31, no. 2, pp. 449-465, 2014.

[18] D. A. Leclair, E. D. Cranston, Z. Xing, and M. R. Thompson, "Evaluation of excipients for enhanced thermal stabilization of a human type 5 adenoviral vector through spray drying," Int. J. Pharm., vol. 506, no. 1-2, pp. 289-301, 2016.

[19] B. C. Hancock and G. Zografi, "The Relationship Between the Glass Transition Temperature and the Water Content of Amorphous Pharmaceutical Solids," Pharm. Res. An Off. J. Am. Assoc. Pharm. Sci., vol. 11, no. 4, pp. 471-477, 1994.

[20] Y. H. Roos and M. Karel, "Plasticizing Effect of Water on Thermal-Behavior and Crystallization of Amorphous Food Models," J. Food Sci., vol. 56, no. 1, pp. 38-43, 1991.

[21] M. J. Pikal, “Freeze Drying,” Enclopedia of Pharmaceutical Technology, no. 713585875. Informa Healthcare USA, Inc., New York City, NY, pp. 1807-1833, 2006.

[22] R. H. Walters, B. Bhatnagar, S. Tchessalov, and K. Izutsu, "Next Generation Drying Technologies for Pharmaceutical,” pp. 2673-2695, 2014.

[23] R. Vehring, "Pharmaceutical particle engineering via spray drying," Pharm. Res., vol. 25, no. 5, pp. 999-1022, 2008.

[24] E. K. Roediger, K. Kugathasan, X. Z. Zhang, B. D. Lichty, and Z. Xing, "Heterologous boosting of recombinant adenoviral prime immunization with a novel vesicular stomatitis virus-vectored tuberculosis vaccine," Mol. Ther., vol. 16, no. 6, pp. 1161-1169, 2008.

[25] A. T. Harding, B. E. Heaton, R. E. Dumm, and N. S. Heaton, "Rationally designed influenza virus vaccines that are antigenically stable during growth in eggs," MBio, vol. 8, no. 3, pp. 1-16, 2017.

[26] P. Corporation, “QuantiFluor ${ }^{\circledR}$ RNA System Technical Manual \#TM377,” p. 19.

[27] J. Oreopoulos, R. F. Epand, R. M. Epand, and C. M. Yip, "Peptide-induced domain formation in supported lipid bilayers: Direct evidence by combined atomic force and polarized total internal reflection fluorescence microscopy," Biophys. J., vol. 98, no. 5, pp. 
815-823, 2010.

574

575

576

577

578

579

580

581

582

583

584

585

586

587

588

589

590

591

592

593

594

595

596

597

598

599

600

601

602

603

604

605

606

607

608

609

610
[28] W. F. Wolkers, A. E. Oliver, F. Tablin, and J. H. Crowe, "A Fourier-transform infrared spectroscopy study of sugar glasses," Carbohydr. Res., vol. 339, no. 6, pp. 1077-1085, 2004.

[29] L. J. Reed and H. Muench, "A Simple method of estimating fifty per cent endpoints," Am. J. Epidemiol., vol. 27, no. 3, pp. 493-497, 1938.

[30] S. Afkhami et al., "Spray dried human and chimpanzee adenoviral-vectored vaccines are thermally stable and immunogenic in vivo," Vaccine, vol. 35, no. 22, pp. 2916-2924, 2017.

[31] J. H. Crowe, A. E. Oliver, F. A. Hoekstra, and L. M. Crowe, "Stabilization of dry membranes by mixtures of hydroxyethyl starch and glucose: the role of vitrification," Cryobiology, vol. 37, no. 1, pp. 20-30, 1998.

[32] D. K. Hincha, E. Zuther, E. M. Hellwege, and A. G. Heyer, "Specific effects of fructoand gluco-oligosaccharides in the preservation of liposomes during drying," Glycobiology, vol. 12, no. 2, pp. 103-110, 2002.

[33] W. F. Tonnis, M. A. Mensink, A. De Jager, K. Van Der Voort Maarschalk, H. W. Frijlink, and W. L. J. Hinrichs, "Size and molecular flexibility of sugars determine the storage stability of freeze-dried proteins," Mol. Pharm., vol. 12, no. 3, pp. 684-694, 2015.

[34] M. Caffrey, V. Fonseca, and A. C. Leopold, "Lipid-Sugar Interactions," Plant Physiol., vol. 86, pp. 754-758, 1988.

[35] J. L. Green and C. A. Angell, "Phase relations and vitrification in saccharide-water solutions and the trehalose anomaly," J. Phys. Chem., vol. 93, no. 8, pp. 2880-2882, 1989.

[36] F. A. Hoekstra, W. F. Wolkers, J. Buitink, E. A. Golovina, J. H. Crowe, and L. M. Crowe, "Membrane stabilization in the dry state," Comp. Biochem. Physiol. - A Physiol., vol. 117, no. 3, pp. 335-341, 1997.

[37] T. H. Jin, E. Tsao, J. Goudsmit, V. Dheenadhayalan, and J. Sadoff, "Stabilizing formulations for inhalable powders of an adenovirus 35-vectored tuberculosis ( TB ) vaccine ( AERAS-402 )," Vaccine, vol. 28, no. 27, pp. 4369-4375, 2010.

[38] P. O. Souillac, C. R. Middaugh, and J. H. Rytting, "Investigation of protein / carbohydrate interactions in the dried state . 2 . Diffuse reflectance FTIR studies," Int. J. Pharm., vol. 235, pp. 207-218, 2002.

[39] D. E. Pegg, "Principles of Cryopreservation," in Cryopreservation and Freeze-Drying Protocols, vol. 368, 2007, pp. 39-57.

[40] M. Bryszewska and R. M. Epand, "Effects of sugar alcohols and disaccharides in inducing the hexagonal phase and altering membrane properties: implications for diabetes mellitus," Biochim. Biophys. Acta, vol. 943, pp. 485-492, 1988. 
Table 1: Glass transition temperatures $\left(\mathrm{T}_{\mathrm{g}}\right)$ of excipients after spray drying (Day 0$)$, and after storage for 7,10 and 15 days at $37^{\circ} \mathrm{C}$ at low relative humidity.

\begin{tabular}{|c|c|c|c|c|}
\hline Sample & $\begin{array}{c}\mathbf{T}_{\mathbf{g}}(\mathbf{D a y} \mathbf{0 , 3 7} \\
\left.{ }^{\circ} \mathbf{C}\right)\end{array}$ & $\begin{array}{c}\mathbf{T}_{\mathbf{g}}(\mathbf{D a y} \mathbf{7 , 3 7} \\
\left.{ }^{\circ} \mathbf{C}\right)\end{array}$ & $\begin{array}{c}\mathbf{T}_{\mathbf{g}}(\mathbf{D a y} \mathbf{1 0}, \mathbf{3 7} \\
\left.{ }^{\circ} \mathbf{C}\right)\end{array}$ & $\mathbf{T}_{\mathbf{g}}(\mathbf{D a y} \mathbf{1 5}$ \\
& 110 & 98 & 72 & $\left.\mathbf{3 7}{ }^{\circ} \mathbf{C}\right)$ \\
\hline Trehalose & 88 & 81 & 86 & 88 \\
\hline Trehalose/Dextran (1:3) & & & & \\
\hline Trehalose/Dextran (3:1) & 88 & 87 & 67 & 61 \\
\hline Mannitol/Dextran (2:1) & 113 & 109 & 101 & 99 \\
\hline Dextran & 125 & 123 & 113 & 107 \\
\hline
\end{tabular}


Figure 1
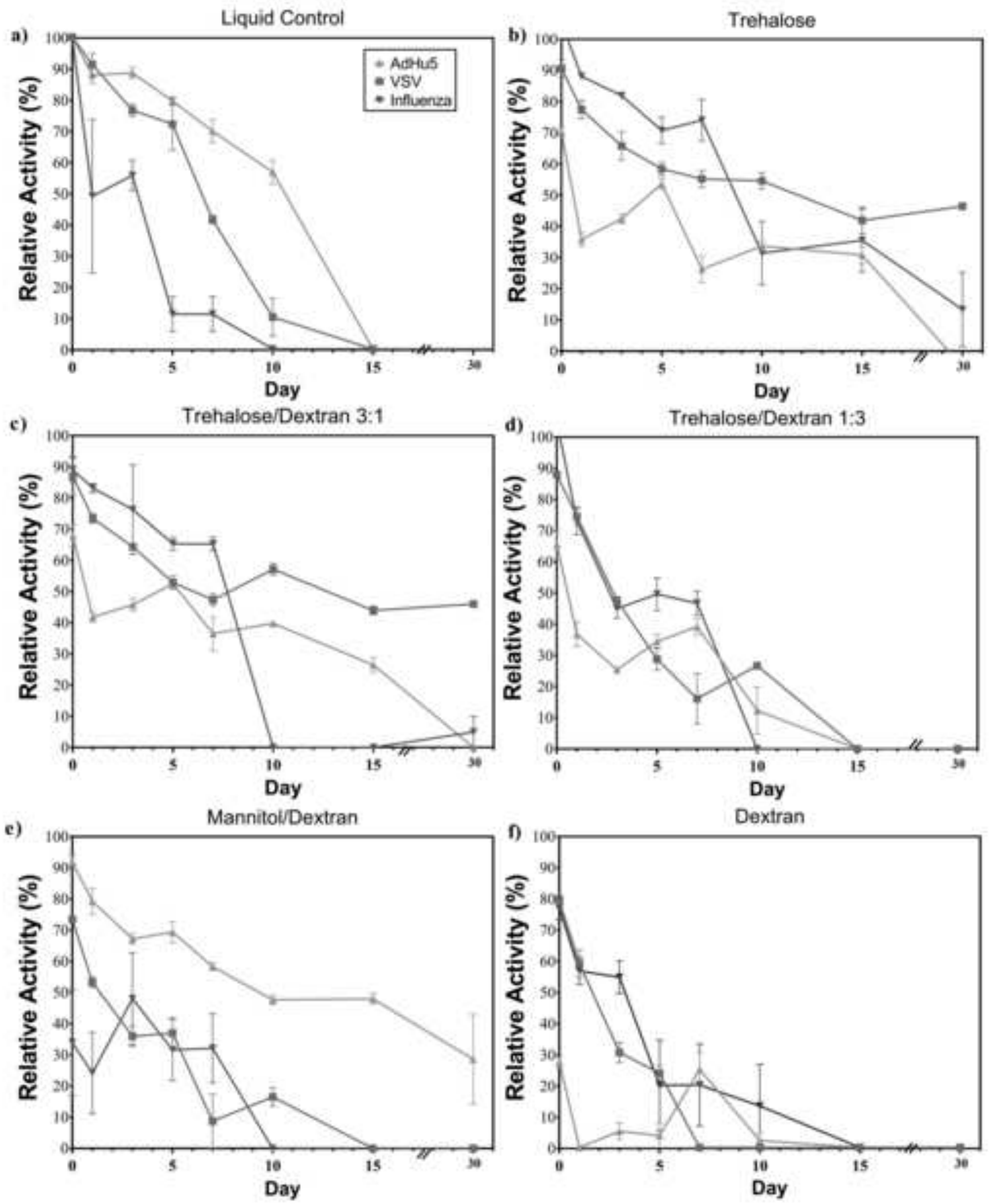
Figure 2

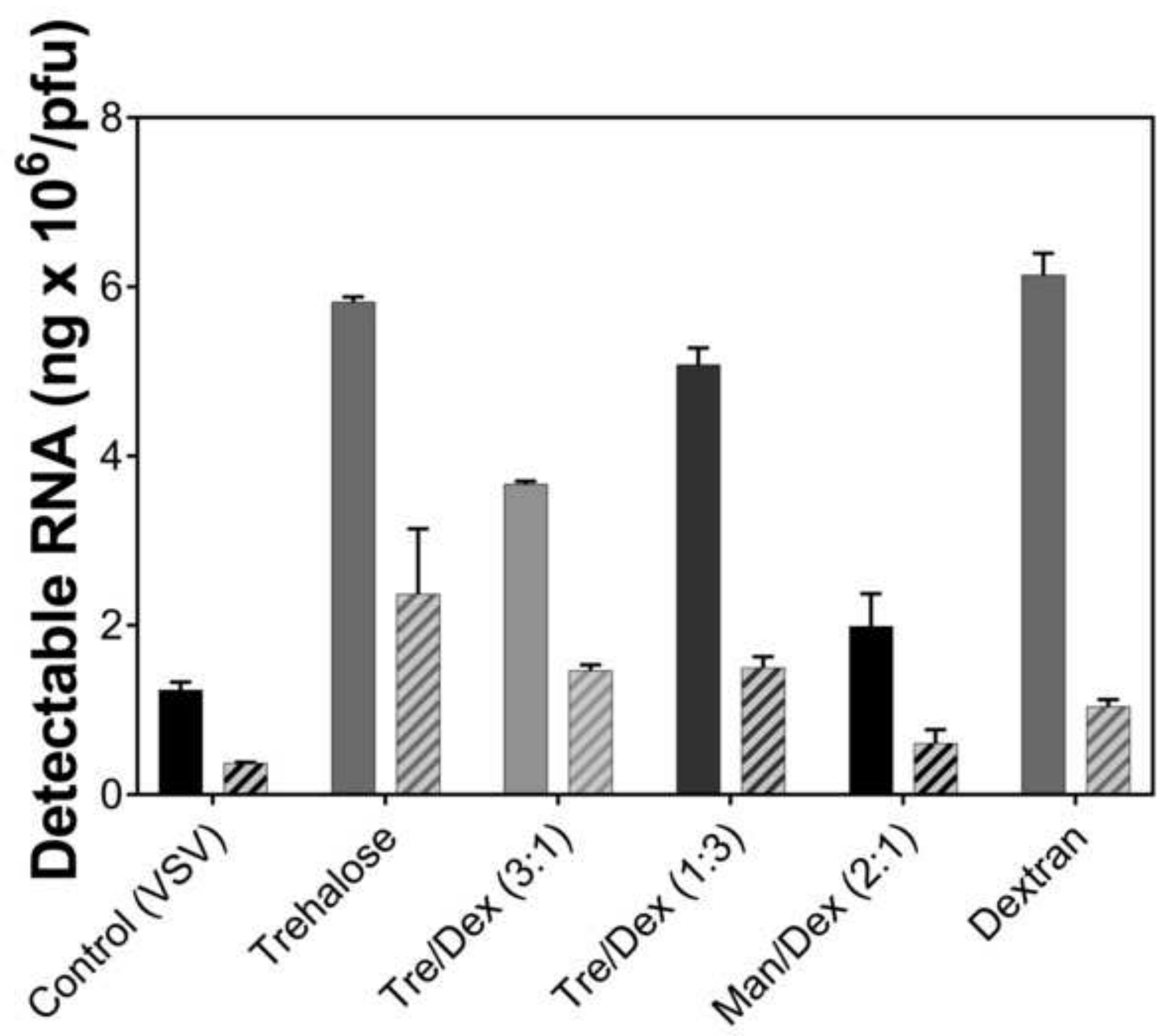




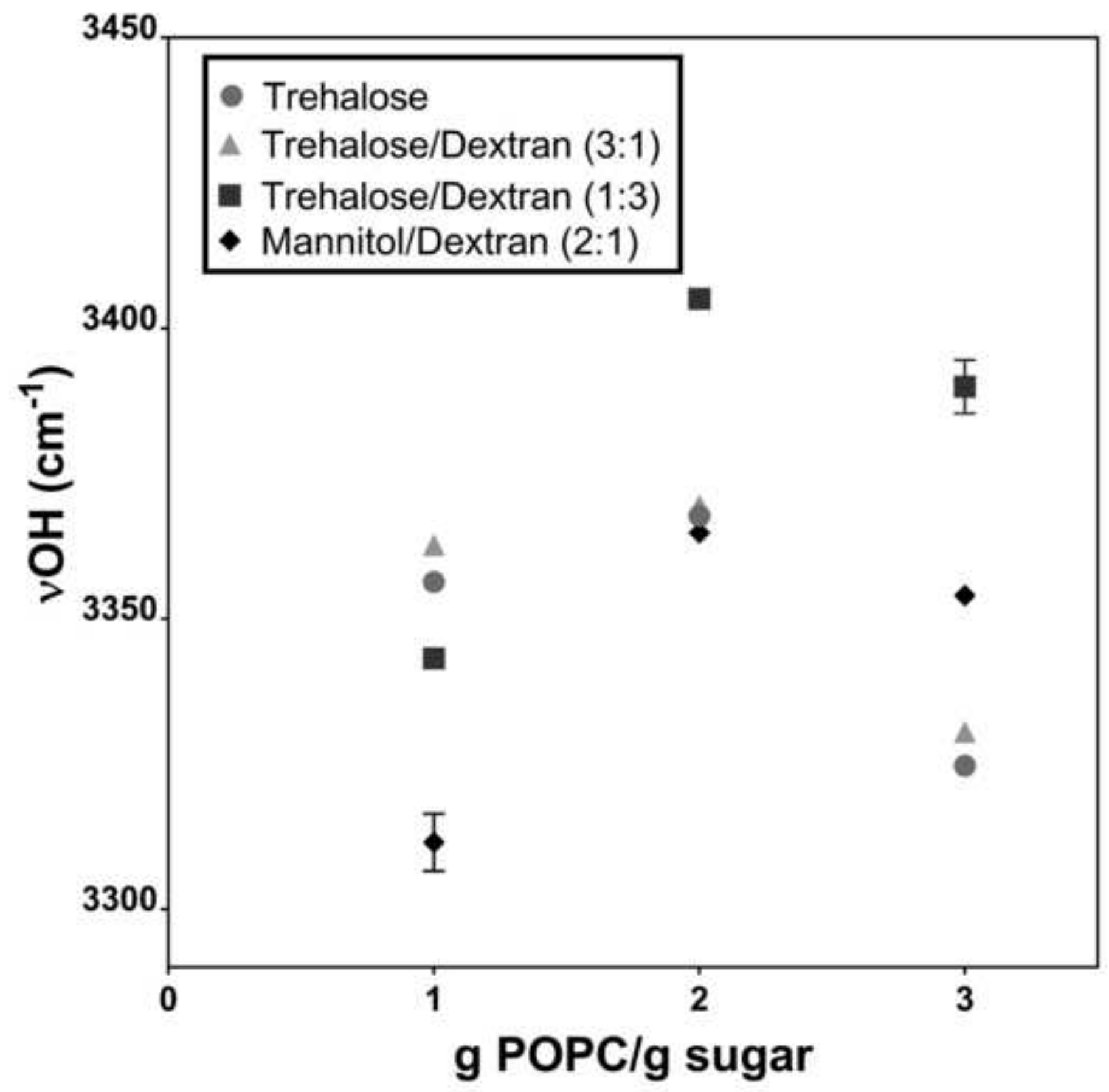

